Our Nature (2003) 1

to perform work. Insurers can still use genetic information in the individual market in decisions about coverage, enrollment, and premiums. Insurers can still require individuals to take genetic tests. Individuals are not protected from the disclosure of genetic information to insurers; plan sponsors (employers), and medical information bureaus, without their consent. Rapid progress in genome science and its potential applications make biology will be the foremost science of the 21st century. Technology and resources generated by the Human Genome Project and other genomics research are already having a major impact on research across the life sciences.

Dil Kumar Subba

Central Campus of Technology, Dharan Emal:dil_subba@hotmail.com

\section{References}

Anonymous, 2002. Human genome project information, Human Genome Program, U. S. Department of Energy Office of Science, Office of Biological and Environmental Research

Fasman, K. H. 2002. Microsoft ${ }^{\circledR} \quad$ Encarta ${ }^{\circledR}$ Encyclopedia.
Jeffords, J. M. and T. Daschle 2001. Genome Era in Political Issues. Science.291 (5507): 1249-1251.

Pääbo, S. 2001. The Human Genome and Our View of Ourselves. Science. 291(5507): 1219-1220.

Wolfsberg, T. G., J Mcentyre and G. D. Schuler 2001. Guide to the draft human genome. Nature 409: 824-826

\title{
2. Avifauna of Tinjure Forest, Eastern Nepal
}

Tinjure Forest, one of the ecologically important regions, lies on the eastern mid-hills of the country at a distance of about $9 \mathrm{~km}$. from Basantapur bazaar of Tehrathum district. Its altitude ranges between 2400-3010 $\mathrm{m}$ and covers 22 sq. km. lying in between Basantapur and Chauki (Rai 1998). Basantapur is a small market located on the boarder of Sankhuvasabha and Terhathum district. Infrastructure of development, such as road and communication has turned this area into the trading centre for Terhathum, Sankhuvasabha and some parts of Bhojpur district. Large amount of Lokta' (Daphne spp.) and medicinal plants are collected there. The area is mostly steep with forested slopes and ridges. The broad-leaved deciduous moist forest mostly covers the area and the lower canopy is composed of Rhododendron spp., Quercus spp., and Daphne spp. It is rich in biodiversity and provided the home to various indigenous and rare birds like Kalij Pheasants
(Lophophora leucomelana) and Satyr Tragopan (Tragopan satyra). There is no specific geographical barrier in between the adjoining areas so that the movement of wildlife and birds within these areas is possible. The area is covered with magnificently beautiful and splendid Rhododendron jungle intermixed mainly with oak and other tree species such as Magnolia spp., Castanopsis spp., Abies spectabilis, Betula utilis, Taxus baccata with different kinds of shrubs, herbs, climbers, epiphytes and grasses. In these regions, survey of bird was conducted in 1997-1998. A total of 73 bird species belonging to 23 families were recorded. Birds were observed in dense forest, degraded forest, bamboo grooves, scrub and open habitats.

Birds were identified (Table 1) with the help of available literature (Fleming et al. 1979, Inskipp and Inskipp 1985, Ali and Ripley 1995). 
Our Nature (2003) 1

Table 1. Checklist of the Birds of Tinjure Forest

\begin{tabular}{|c|c|c|c|c|c|}
\hline S. N. & English Name & Scientific Name & Family & S1 & Forest Type \\
\hline 1. & Black Kite & Milvus migrans migrans & Accipritidae & 2 & $\mathrm{O}$ \\
\hline 2. & Crested serpent Eagle & Spilornis chella & $"$ & 1 & $\mathrm{OT}$ \\
\hline 3. & Besra Sparrowhawk & Accipiter virgatus & $"$ & 4 & $\mathrm{~T}$ \\
\hline 4. & Common Buzzard & Buteo buteo & $"$ & 2 & OT \\
\hline 5. & Black Eagle & Ictinaetus malayensis & $"$ & 2 & $\mathrm{D}$ \\
\hline 6 & $\begin{array}{l}\text { Himalayan Griffon } \\
\text { Vulture }\end{array}$ & Gyps himalayensis & " & - & $\mathrm{O}$ \\
\hline 7 & Steppe Eagle & Aquila nipalensis & " & 2 & $\mathrm{O}$ \\
\hline 8 & Impeyan Pheasant & Lophophorus impejanus & Phasianidae & - & $\mathrm{O}$ \\
\hline 9 & Satyr Tragopan & Tragopan satyra & " & - & $\mathrm{D}$ \\
\hline V10 & Kalij Phesant & Lophophora leucomelana & $"$ & 2 & $\mathrm{D}$ \\
\hline 11 & Rufous Turtle Dove & Streptopelia orientalis & Columbidae & 1 & TD \\
\hline 12 & Large-Hawk Cuckoo & Cuculus sparverioides & Cuculidae & 2 & $\mathrm{D}$ \\
\hline 13 & Collared Pigmy Owlet & Glaucidium brodiei & Strigidae & - & $\mathrm{T}$ \\
\hline V14 & Brownwood Owlet & Strix leptogrammica & $"$ & 5 & $\mathrm{D}$ \\
\hline 15 & $\begin{array}{l}\text { White-throated } \\
\text { Needle tail Swift }\end{array}$ & Chaetura caudacuta & Apodidae & 5 & $\mathrm{O}$ \\
\hline 16 & $\begin{array}{l}\text { Large White-rumped } \\
\text { Swift }\end{array}$ & Apus pacificus & $"$ & - & $\mathrm{O}$ \\
\hline 17 & Hoopoe & Upupa epops & Upupidae & - & $\mathrm{OT}$ \\
\hline 18 & $\begin{array}{ll}\text { Great } & \text { Himalayan } \\
\text { Barbet } & \\
\end{array}$ & Megalaima virens & Capitonidae & - & DT \\
\hline 19 & Blue- throated Barbet & Megalaima asiatica & $"$ & 2 & DT \\
\hline 20 & $\begin{array}{ll}\text { Darjeeling } & \text { Pied } \\
\text { Woodpecker } & \\
\end{array}$ & $\begin{array}{l}\text { Dendrocopos } \\
\text { darjellensis }\end{array}$ & Picidae & 1 & $\mathrm{D}$ \\
\hline 21 & Barn Swallow & Hirundo rustica & Hirundinidae & 1 & $\mathrm{O}$ \\
\hline 22 & Nepal House Martin & Delichon nipalensis & $"$ & 2 & TO \\
\hline 23 & Hodgson's Tree Pipit & Anthus hodgsoni & Motacillidae & 1 & $\mathrm{O}$ \\
\hline 24 & Upland Pipit & Anthus sylvanus & $"$ & 2 & $\mathrm{O}$ \\
\hline 25 & Pied Wagtail & Motacilla alba & $"$ & - & $\mathrm{O}$ \\
\hline 26 & Scarlet Minivet & Pericrocotus flammeus & $\begin{array}{l}\text { Campephagi } \\
\text { dae }\end{array}$ & 2 & TD \\
\hline 27 & Short-billed Minivet & $\begin{array}{l}\text { Pericrocotus } \\
\text { brevirostris }\end{array}$ & " & - & TD \\
\hline 28 & Long-tailed Minivet & Pericrocotus ethlogus & " & 1 & TD \\
\hline 29 & White-cheeked Bulbul & Pycnonotus leucogenys & Pycnonotidae & 1 & TS \\
\hline 30 & Common Stonechat & Saxicola torquata & Muscicapidae & 2 & $\mathrm{SO}$ \\
\hline 31 & Dark Gray Bushchat & Saxicola ferrea & $"$ & 2 & $\mathrm{SO}$ \\
\hline 32 & $\begin{array}{l}\text { Chestnut-bellied } \\
\text { Rock Thrush }\end{array}$ & Monticola rufiventris & " & - & DT \\
\hline 33 & Blue Whistling Thrush & Myiophonus caeruleus & $"$ & 3 & DT \\
\hline+34 & $\begin{array}{l}\text { Plain-backed } \\
\text { Mountain Thrush }\end{array}$ & Zoothera mollissima & " & 4 & $\mathrm{D}$ \\
\hline
\end{tabular}


Our Nature (2003) 1

\begin{tabular}{|c|c|c|c|c|c|}
\hline+35 & $\begin{array}{ll}\text { White- } & \text { collared } \\
\text { Blackbird } & \end{array}$ & Turdus albocinctus & " & 2 & $\mathrm{D}$ \\
\hline+36 & Gray-winged Blackbird & Turdus boulboul & " & 2 & $\mathrm{D}$ \\
\hline+37 & $\begin{array}{l}\text { Rufous-capped Bush } \\
\text { Warbler }\end{array}$ & Cettia brunnifrons & " & 2 & SD \\
\hline 38 & Black-browed Warbler & Seicercus burkii & $"$ & 1 & DTS \\
\hline 39 & $\begin{array}{l}\text { Chesnut- crowned } \\
\text { warbler }\end{array}$ & Seicercus castaniceps & " & 1 & $\mathrm{D}$ \\
\hline 40 & Gray-headed Warbler & Seicercus xanthochistos & " & 1 & $\mathrm{D}$ \\
\hline 41 & $\begin{array}{l}\text { Gray-faced Leaf } \\
\text { Warbler }\end{array}$ & $\begin{array}{l}\text { Phylloscopus } \\
\text { maculipennis }\end{array}$ & " & 1 & $\mathrm{D}$ \\
\hline 42 & Verditer Flycatcher & Muscicapa thalassina & " & 1 & $\mathrm{~T}$ \\
\hline 43 & Asian sooty Flycatcher & Muscicapa sibirica & " & 1 & $\mathrm{~T}$ \\
\hline V44 & $\begin{array}{l}\text { White- } \\
\text { Flycatcher }\end{array}$ & Muscicapa monileger & " & 4 & $\mathrm{D}$ \\
\hline+45 & $\begin{array}{l}\text { Black-faced } \\
\text { Laughing Thrush }\end{array}$ & Garrulax affinis & " & 1 & $\mathrm{D}$ \\
\hline 46 & Hoary Barwing & Actinodura nipalensis & " & 2 & $\mathrm{D}$ \\
\hline+47 & Bar-throated Minla & Minla strigula & " & 1 & $\mathrm{D}$ \\
\hline 48 & Red-tailed Minla & Minla ignotincta & " & 2 & $\mathrm{D}$ \\
\hline 49 & $\begin{array}{l}\text { Chestnut-headed Tit } \\
\text { Babbler }\end{array}$ & Alcippe castaneceps & " & 1 & $\mathrm{D}$ \\
\hline+50 & $\begin{array}{l}\text { White-browed Tit } \\
\text { Babbler }\end{array}$ & Alcippe vinipectus & " & 1 & DT \\
\hline+51 & Black-capped Sibia & Heterophasia capistrata & " & 1 & DT \\
\hline 52 & Yellow-napped Yuhina & Yuhina flavicollis & " & 1 & $\mathrm{D}$ \\
\hline 53 & Stripe-throated Yuhina & Yunina gularis & " & 1 & $\mathrm{D}$ \\
\hline+54 & Rufous-vented Yuhina & Yunina occipitalls & " & 1 & TD \\
\hline 55 & Red-headed Tit & Aegithalos concinnus & Paridae & 1 & TD \\
\hline+56 & Rufous-bellied Black Tit & Parus rubidiventris & & 1 & $\mathrm{D}$ \\
\hline 57 & Green-backed Tit & Parus monticolus & " & 1 & DT \\
\hline 58 & Yellow-cheeked Tit & Parus xanthogenys & $"$ & - & DTS \\
\hline 59 & Nepal Sunbird & Aethopyga nipalenis & Nectariniidae & 1 & DS \\
\hline 60 & Oriental White eye & Zosterops palpebrosa & Zosteropidae & 1 & TS \\
\hline 61 & Black-headed Shrike & Lanius schach & Daniidae & 1 & OS \\
\hline 62 & Gray-backed Shrike & Lanius tephronotous & " & 2 & OS \\
\hline 63 & Bronzed Drongo & Dicrurus aeneus & Dicruridae & 3 & $\mathrm{~T}$ \\
\hline 64 & Black Drongo & Dicrurus adsimilis & " & - & TO \\
\hline 65 & Ashy Drongo & Dicrurus leucophaeus & " & - & $\mathrm{T}$ \\
\hline+66 & $\begin{array}{l}\text { Yellow-billed Blue } \\
\text { Magpie }\end{array}$ & Cissa flavirostris & Corvidae & 1 & $\mathrm{D}$ \\
\hline 67 & Himalayan Treepie & Dendrocitta formosae & $"$ & 1 & $\mathrm{~T}$ \\
\hline 68 & Nutcracker & Nucifraga caryocatactes & " & - & DT \\
\hline 69 & Jungle Crow & Corvus macrorhynchos & " & 1 & TO \\
\hline 70 & Tree Sparrow & Passer montanus & Ploceidae & - & $\mathrm{T}$ \\
\hline
\end{tabular}


Our Nature (2003) 1

\begin{tabular}{|c|l|l|l|c|c|}
\hline 71 & Common Rosefinch & Carpodacus erythrinus & Fringillidae & - & ST \\
\hline 72 & Red-headed Bullfinch & Pyrrhula erythrocephala & $"$ & 1 & D \\
\hline 73 & Little Bunting & Emberiza pusilla & Emberizidae & 2 & OTS \\
\hline
\end{tabular}

$\begin{array}{llllll}\text { Note: } & & & & \\ 1 & = & \text { Common TO } & = & \text { Thin / Open } \\ 2 & = & \text { Fairly common } & \mathrm{T} & = & \text { Thin } \\ 3 & = & \text { Occasional } & \text { ST } & = & \text { Scrub / Thin } \\ 4 & = & \text { Uncommon } & \text { D } & = & \text { Dense } \\ 5 & = & \text { Rare } & \text { OTS } & & \text { Open / Thin / Scrub } \\ \mathrm{S} 1 & = & \text { Status } & & & \\ + & = & \text { Internationally significant breeding population }\end{array}$

Mala Rai

Central Department of Zoology Tribhuvan University, Kathmandu Corresondence: Department of Biology, Central Campus of Technology, Hattisar, Dharan

\section{References}

Ali, S. and S. D. Ripley 1995. A Pictorial Guide to the Birds of Indian Subcontinent. Bombay Natural History Society, Oxford University Press, Bombay

Fleming, R. L (Sr)., R. L. Fleming (Jr) and L. S. Bangel 1979. Birds of Nepal, Second Edition, Avalok Publishers, Kathmandu, Nepal
Inskipp, C and T. Inskipp 1985. A Guide to the Birds of Nepal. Croom Helm, London, England

Rai, M. 1998. The impact of development activities on Avifauna of Tamor river basin" M. Sc. Thesis, Central Department of Zoology, T. U., Kirtipur Kathmandu.

\section{Birds of Itahari}

Itahari municipality is one of the three municipalities of Sunsari district. It is situated in the Tarai region of Eastern part of Nepal. The main rivers are Sunsari, Tengra and Budhi. Besides that there are also few man-made and natural wetlands. Itahari is very near from the mixed type of jungle popularly known as 'Charkoshe Jhadi' on the north, which provides ideal shelter for the birds. This is the reason why many local migratory birds as well as other birds are found to come very commonly from the jungle.
It was the month of November 2000 when I went to Itahari to watch birds for the first time. Then I kept watching the birds at that place regularly till January 2001. During that period I visited different sites several times at least once a week regularly and also collected information from the locals.

The present report is the result of a study of about 14 months. Regular observations and local enquiry were also made. I tried my best to identify the birds correctly using available literature (Ali 